\title{
Towards the Next Generation of Service-Based Systems: The S-Cube Research Framework*
}

\author{
Andreas Metzger and Klaus Pohl \\ Software Systems Engineering, University of Duisburg-Essen \\ 45117 Essen, Germany \\ \{andreas.metzger, klaus.pohl\}asse.uni-due.de
}

\begin{abstract}
Research challenges for the next generation of service-based systems often cut across the functional layers of the Service-Oriented Architecture (SOA). Providing solutions to those challenges requires coordinated research efforts from various disciplines, including service engineering, service composition, software engineering, business process management, and service infrastructure. The FP7 Network of Excellence on Software Services and Systems (S-Cube) performs cross-discipline research to develop solutions for those challenges. Research in S-Cube is organised around the S-Cube research framework, which we briefly introduce in this paper. Moreover, we outline the envisioned types of interactions between the key elements of the S-Cube research framework, which facilitate the specification and design as well as the operation and adaptation of future service-based systems.
\end{abstract}

Keywords: Service-based Systems, Service Oriented Architecture, Service Engineering, Software Services.

\section{Motivation}

Service-orientation is increasingly adopted as a paradigm for building highly dynamic, distributed and adaptive software systems, called service-based systems. A service-based system is realized by composing software services. For the service composer, a software service is not an individual piece of software. Rather, it represents some functionality that can be invoked through the service's interface, where the actual software that implements this functionality is executed, maintained and owned by the provider of that service [1].

Currently, the common practice for developing service-based systems is to employ the Service-Oriented Architecture (SOA) paradigm, which distinguishes between three functional layers [2][3][4]: The service infrastructure layer (SI) supports describing, publishing and discovering services and provides the run-time environment for the execution of service-based systems. It provides primitives for service communication, facilities for service description, as well as capabilities for service discovery.

* The research leading to these results has received funding from the European Community's Seventh Framework Programme FP7/2007-2013 under grant agreement 215483 (S-Cube). For further information please visit http://www.s-cube-network.eu/ 
The service composition and coordination layer (SCC) supports the (hierarchical) composition of multiple services. Such service compositions can in turn be offered to service clients, used in further service compositions and eventually be composed to service-based systems. The business process management layer (BPM) provides mechanisms for modelling, analysing and managing business processes that can span the administrative boundaries of multiple organizations.

When building service-based systems one typically faces challenges which cut across the functional SOA layers, such as:

- Incomplete knowledge: A service-based system cannot be specified completely in advance due to the incomplete knowledge about the interacting parties (e.g., service providers) as well as the system's context. Thus, compared to traditional software engineering, much more decisions need to be taken during the run-time (operation) phase. As a consequence, new life cycle models and methods will have to support the agility, flexibility and dynamism required to leverage the potential of service-based systems.

- Adaptations across the functional layers: Adaptations at the functional layers can be conflicting. For example, the service composition layer might perform a fragmentation of the business process while at the same time the infrastructure layer might initiate a data fragmentation. These two adaptations can be in conflict if, for example, the data required by a process fragment is moved to another location than the process fragment, or if the data fragmentation leads to a violation of privacy policies stipulated by the process fragments. Thus, an adaptation strategy is required which coordinates adaptations across the functional layers and thereby avoids such conflicts.

- End-to-end-quality: Each functional layer contributes to the end-to-end quality of a service-based system. Thus, to assure end-to-end quality, the different quality characteristics (like reliability or performance) and their dependencies must be understood and the different quality levels, as stipulated in individual quality contracts (e.g., as part of SLAs), need to be aggregated across the functional layers.

Addressing such cross-cutting challenges (see [5][6] for a more detailed list) requires an integration of knowledge and competencies of various disciplines, including service engineering, service composition and orchestration, software engineering, business process management, and service infrastructure. In Section 2 we sketch the $\mathrm{S}$-Cube research framework, which provides a clear separation of concerns but also systematically addresses the cross-cutting challenges. In Section 3 we outline the envisioned types of interactions between the key elements of the S-Cube framework, which facilitate the specification and design as well as the operation and adaptation of future service-based systems.

\section{The S-Cube Research Framework}

Figure 1 provides an overview of the S-Cube research framework and its key elements. In addition to the functional SOA layers (BPM, SCC and SI - in the S-Cube framework we call those layers technology layers), the S-Cube research framework introduces the following cross-cutting elements [7]: 
- Service engineering and design (SED): SED provides principles, techniques, methods and life-cycle models which facilitate the creation of innovative servicebased systems, which include requirements engineering and design principles and techniques. SED takes a holistic view on engineering, operating and evolving service-based systems.

- Service adaptation and monitoring (SAM): SAM facilitates the cross-layer monitoring of service-based systems as well as their continuous adaptation in response to, e.g., context changes, system failures or underperformance. In addition, SAM supports the pro-active adaptation of service-based systems across the three layers.

- Service quality definition, negotiation and assurance (SQDNA): SQDNA provides principles, techniques and methods for defining, negotiating and assuring end-toend quality of service-based systems as well as conformance to SLAs. In addition, it facilitates pro-active adaptations by providing novel techniques for predicting the future quality of service-based systems.

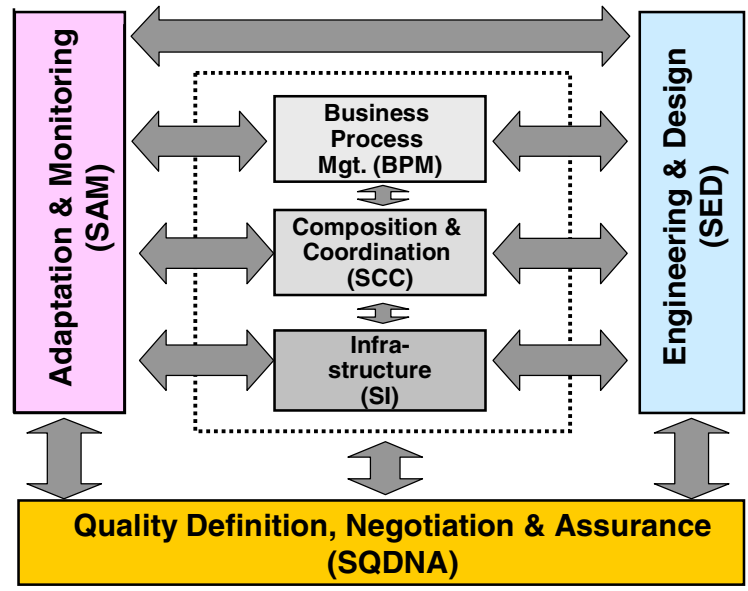

Fig. 1. Overview on the S-Cube research framework

\section{Envisioned Interactions between the Framework Elements}

For each element of the S-Cube research framework, its interactions and interfaces with the other framework elements are defined. We distinguish between two principle kinds of interactions:

- Design and Specification Interactions: These interactions expose the capabilities and features of one framework element to another framework element. The exposed capabilities and features are taken into account when engineering, designing, monitoring, adapting or assuring the quality of a service-based system. Moreover, the cross-cutting elements (SED, SAM and SQNDA) can constrain the capabilities offered by the three technology layers by specifying how to use those capabilities in a concrete service-based system. 
- Operation and Run-time Interactions: These interactions reflect the information which is exchanged between the framework elements during the operation, execution and adaptation of the service-based system as well as its instances.

We illustrate the two types of interactions by describing the envisioned interactions between the SED, SAM and SCC elements depicted in Figure 2.

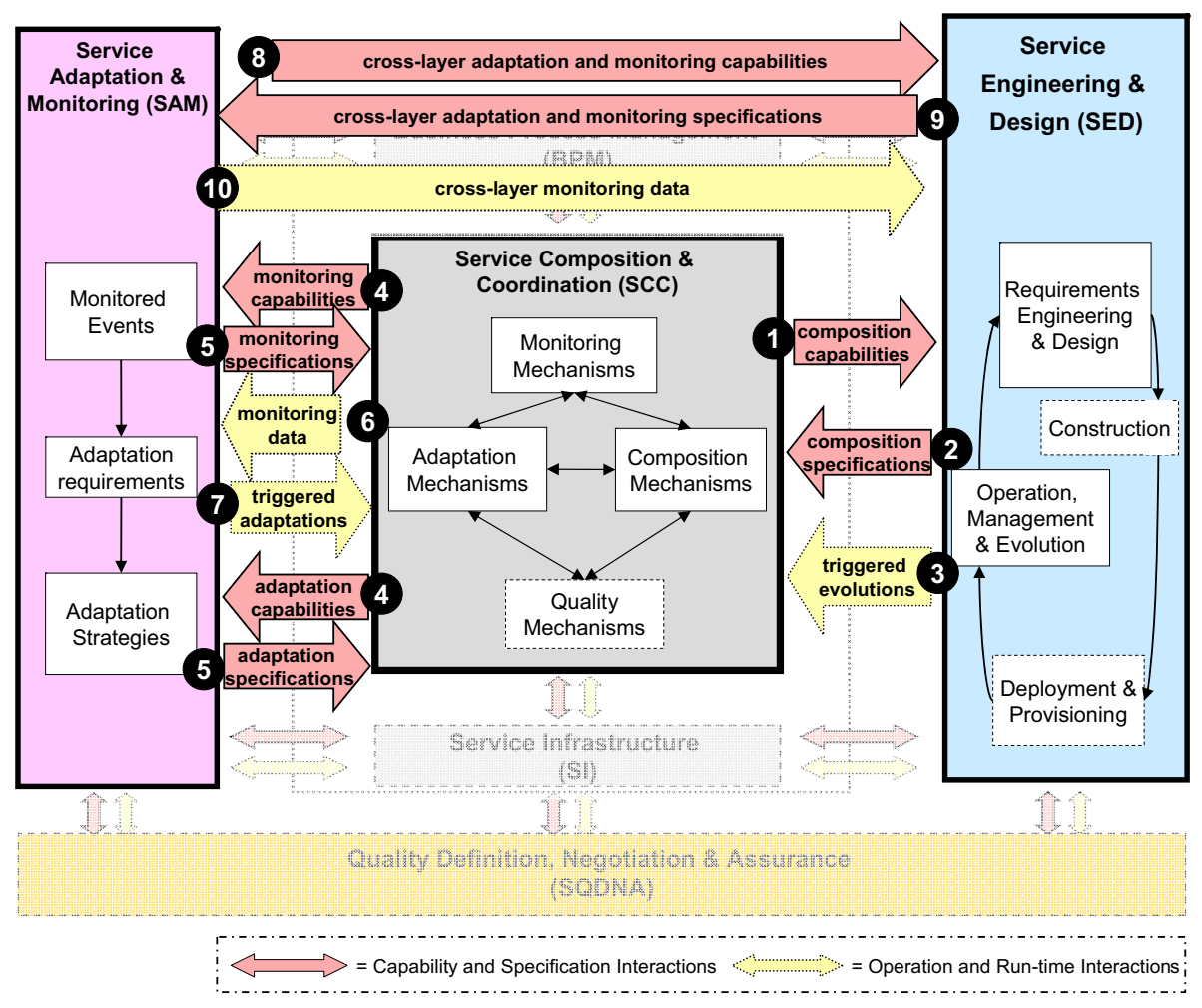

Fig. 2. Envisioned interactions between the SAM, SED and SCC elements

\section{Envisioned interactions between SCC and SED}

As depicted in Figure 2 we envision three principle interactions between SCC and SED:

(1) The SCC layer communicates its composition capabilities to the SED element. The SED element exploits those capabilities during requirements engineering and design of the service-based system together with the capabilities of the SI, BPM, SAM and SQDNA elements.

(2) As a result of the engineering and design activities, the SED element specifies which SCC capabilities should be used for the service-based system at hand. The SED element communicates those specifications to the SCC layer. It thereby can restrict the capabilities offered by the SCC layer. For example, the SED element can forbid the use of a certain composition mechanism or define the order in 
which the mechanisms have to be used. In addition, the SED element also communicates the specifications for the quality, the monitoring and the adaptation capabilities to the SCC layer (not depicted in Figure 2).

(3) In the case of an evolution of the service-based system, the SED element communicates the evolution triggers (i.e., actions to be executed to implement the evolution) to the SCC layer. This includes updates of the specifications for the SCC capabilities.

\section{Envisioned interactions between SAM and SCC}

Between SCC and SAM the following four key interactions are envisioned:

(4) The SCC layer communicates its capabilities to collect monitoring data from the service compositions to the SAM element. In addition, the SCC layer exposes its capabilities for adapting service compositions to the SAM element. The SAM element uses those capabilities within its cross-layer adaptation techniques.

(5) Based on the monitoring and adaptation strategies designed for the service-based system, the SAM element communicates the monitoring and adaptation specification for the service-based system at hand to the SCC layer and thereby defines which capabilities are valid for that service-based system.

(6) During the operation of the service-based system, the collected monitoring data is communicated from the SCC layer to the SAM element. The SAM element analyzes the monitoring data received - under consideration of the monitoring data received from the SI and BPM layers - and determines required adaptations.

(7) The adaptation strategies of the SAM element ensure conflict-free, cross-layer adaptations. If adaptations of the service compositions are required, the SAM element communicates the required adaptation specifications to the SCC layer.

\section{Envisioned interactions between SAM and SED}

As shown in Figure 2, three principle interactions between SAM and SED are envisioned:

(8) The SAM element communicates its cross-layer monitoring and adaptation capabilities to the SED element, which considers those capabilities during the engineering and design of the service-based system. For example, the SED element could decide whether to use intrusive monitoring or non-intrusive monitoring in a certain system and how the monitoring capabilities of all three technology layers should be correlated.

(9) The SED element communicates the cross-layer adaptation and monitoring specification for the service-based system at hand to the SAM element. For example, the specification can define that only non-intrusive monitoring capabilities are to be used by all three technology layers. The SAM element follows the specification and adjusts his monitoring and adaptation capabilities for the service-based system accordingly.

(10) During the operation of the service-based system, the SAM element correlates the monitoring data from the different layers and provides correlated, cross-layer monitoring data to the SED element. This data is analyzed to determine if an evolution of the service-based system is required (e.g., in the case that adaptations of individual instances of the service-based system do not suffice). 
The small arrows in Figure 2 indicate interactions between framework elements which are not described above. The $\mathrm{S}$-Cube research vision white paper describes all envisioned interactions of the S-Cube research framework. The white paper is available from the S-Cube web portal at http://www.s-cube-network.eu/.

\section{Conclusions}

The FP7 Network of Excellence S-Cube addresses the cross-cutting research challenges faced when engineering, designing, adapting, operating and evolving the next generation of service-based systems. S-Cube's research is guided by the research framework and its envisioned interactions sketched in this paper. The S-Cube research framework clearly distinguishes between principles and methods for engineering and adapting service-based systems and the technology and mechanisms which are used to realize those systems, while taking into account cross-cutting issues like Quality of Service (QoS) and SLA compliance. By synthesizing and integrating diversified knowledge across different research disciplines, S-Cube develops the next generation of principles, techniques and methods for the service-based systems of the future.

Acknowledgments. We cordially thank all S-Cube members for the fruitful discussions and their contributions to the S-Cube research framework.

\section{References}

[1] Di Nitto, E., Ghezzi, C., Metzger, A., Papazoglou, M., Pohl, K.: A Journey to Highly Dynamic, Self-adaptive Service-based Applications. Automated Software Engineering 15(3-4) (December 2008)

[2] Erl, T.: Service-oriented Architecture. Prentice-Hall, Englewood Cliffs (2004)

[3] Josuttis, N.: SOA in Practice: The Art of Distributed System Design. O'Reilly, Sebastopol (2007)

[4] Kaye, D.: Loosely Coupled: The Missing Pieces of Web Services. RDS Press (2003)

[5] Papazoglou, M., Pohl, K.: S-Cube: The Network of Excellence on Software Services and Systems. In: Di Nitto, E., Traverso, P., Sassen, A., Zwegers, A. (eds.) At Your Service: An Overview of Results of Projects in the Field of Service Engineering of the IST Programme. MIT Press Series on Information Systems (2009)

[6] Papazoglou, M., Pohl, K.: Report on Longer Term Research Challenges in Software and Services. In: Boniface, M., Ceri, S., Hermenegildo, M., Inverardi, P., Leymann, F., Maiden, N., Metzger, A., Priol, T. (eds.) Results from two workshops held at the European Commission premises at 8th of November 2007 and 28th and 29th of January 2008, European Commission (2008), http: / / www . cordis. Iu

[7] Metzger, A., Pohl, K.: S-Cube: Enabling the Next Generation of Software Services. In: Filipe, J., Cordeiro, J., Cardoso, J. (eds.) Proceedings of the 5th Intl. Conference on Web Information Systems and Technologies (WEBIST 2008). LNBIP, vol. 18, pp. 40-47. Springer, Heidelberg (2009) 